\title{
Poésie et allégorie : \\ Claudel et « La muraille intérieure de Tokyo »
}

\author{
Sergio Villani \\ York University
}

\section{Cette communication focalise l'attention sur « La} muraille intérieure de Tokyo », publié en 1922, un poème de la période japonaise de Claudel. Adoptant une perspective intertextuelle, j'analyse ce poème dans le contexte d'une suite d'écrits claudéliens publiés entre 1918 et 1923, notamment le poème "Sainte Geneviève », commence en 1918 au Brésil et achevé au Japon en 1923; "L'ode jubilaire ", un poème de circonstance qui célèbre le $600^{\circ}$ anniversaire de la mort de Dante, publié en 1921; et « La route interrompue » publié en 1923. Ces poèmes ${ }^{1}$ sont associés par la proximité temporelle de leur genèse et publication mais aussi par les thèmes du voyage, de la promenade et de la représentation, aussi bien que par les images du mur, du miroir et de la route. Certains de ces motifs et images s'annoncent dans les premiers vers écrits par Claudel. "La Maison intérieure de Tokyo » reprend ces images et motifs, les amplifie et les relie, tout en faisant résonner leur polysémie.

L'intention de Claudel dans ces textes et en particulier dans «La muraille intérieure de Tokyo » est double : Définir une vision existentielle et en même temps définir une poétique de la représentation. Pour ce faire il articule un discours compose d'analogies et qui comporte deux niveaux de signification, l'un littéral, l'autre symbolique, l'un temporel, l'autre intemporel. En fait, Claudel pratique une écriture allégorique stimulée à ce temps en partie par sa méditation au début des années 20 sur l'œuvre et la figure de Dante, un frère poète, pèlerin, qui connût comme lui, les chemins de l'exil. 


\section{Sergio Villani}

Claudel conçoit sa vie comme une ligne fragmentée, interrompue et reprise, et représentée surtout pas la genèse de ses œuvres qui manifestent aussi une suite d'interruptions et de reprises. C'est ainsi qu'en rédigeant « La Muraille intérieure de Tokyo » au verso des feuilles où il écrit sa «Sainte Geneviève », il évoque son séjour au Brésil où la composition de «Sainte Geneviève " prit sa première forme, fut par la suite interrompue avant d'être reprise des années plus tard au Japon. Cette méditation sur Sainte Geneviève est une tentative à l'étranger de se relier a une image sainte, symbole de son pays, de son peuple et de son histoire temporelle et sacrée. En lisant «L'Ode jubilaire » et « La Route interrompue », on a l'impression que Claudel est déconcerté par son existence fragmentée, faite d'une suite de déracinements et de déplacements. Pourtant, on ressent aussi une certaine résignation, une consolation qu'il dérive d'images comme celle de Geneviève et de Dante, et, comme le suggère « La route interrompue ", de sa foi que toutes ses pérégrinations font partie d'un dessein providentiel :

Le chemin qu'à travers l'œuvre de Dieu promène l'explorateur sans cesse retrouvé et reperdu.

Et dont j'essaye en vain bout à bout de rejoindre les fragments interrompus (OP, p. 694)

Au début des années 20, à la suite de la Grande Guerre, la pensée européenne semble sombrer dans le noir. En France, Paul Valéry, par exemple, élabore dans ces essais l'idée d'une «crise de l'esprit », une «crise de l'intelligence. » Et le jeune Malraux, imbu de ses lectures de Nietzsche, médite le crépuscule de la civilisation occidentale. Par contre, malgré l'aspect fragmentaire de sa vie à l'étranger, Claudel s'efforce d'exprimer une sérénité, une confiance et un espoir que lui assure la pratique de sa foi et de son art.

«La muraille intérieure de Tokyo " est une méditation existentielle et esthétique développée à travers des images et un récit qui forment une allégorie. Portons notre attention d'abord sur la 
Poésie et allégorie : « La muraille intérieure de Tokyo »

première et a dernière partie où domine l'image du mur ou de la muraille annoncée dans le titre du poème :

Non point la forêt ni la grève, chaque jour le site de ma promenade est un mur,

Il y a toujours un mur à ma droite.

Un mur que je suis et qui me suit et que je déroule derrière moi en marchant et devant moi il y en encore provision et fourniture.

Un mur continuellement à ma droite.

A ma gauche il y a la ville et les grandes avenues en partance vers toute la terre.

Mais il y a un mur à ma droite.

Je tourne (à cette station du tram) et je sais que c'est par là la mer,

Mais le mur est indécollable à ma droite,

Il y a toute une ville sous mes pieds, tout un monde fragile dans le soir qui s'allume et qui s'éteint,

Mais cela n'empêche pas ce mur à ma droite, Un mur qui ne me conduit ailleurs que pour me ramener au même point,

Et quand je fermerais les yeux, je n'ai qu'à tendre la main Pour vérifier cette présence à ma droite. [...]

\section{XII}

J'habite l'extérieur d'un anneau.

J'ai appris que ce n'est pas dehors, c'est dedans qu'est le mur dont je suis le prisonnier.

J'ai appris que pour aller d'un point à un autre il est possible de passer partout excepté par le centre. (OP, p. 646-650) 
La répétition de l'expression "j'ai appris », à la fin du poème, nous indique que Claudel veut communiquer un savoir, une connaissance acquise par son expérience existentielle, religieuse et artistique. Cependant, l'expression de cette connaissance ici demeure sinon énigmatique, mystique et il faut donc faire appel à une certaine herméneutique ou à une lecture proche $d u$ texte afin de la déchiffrer et la comprendre.

"Le paysage a un sens ", écrit Claudel dans « la Route interrompue » (OP, p. 692). Le monde n'est pas absurde pour lui, car le monde est l'image (ou le reflet) du Créateur, de Dieu. La Nature n'est pas seulement le Temple de Baudelaire, image païenne, et sa "forêt de symboles ", mais pour Claudel une " cathédrale », la maison de Dieu, un espace catholique, intemporel et cosmique. De cette perspective, tout a un sens. Quel serait donc le sens du paysage de "la Muraille intérieure de Tokyo "?

Le paysage dans ce poème est réaliste pour la plupart, et désigne une ville, Tokyo : il y a les grandes avenues, les lumières du soir, la station de tram, la mer, et la muraille, site de la promenade quotidienne du poète. Toute l'attention est dirigée sur ce mur qui est toujours à sa droite. "Continuellement ", "indécollable », écrit-il. Ce mur est une présence incontournable, indéniable, qui le rassure et le ramène toujours au point de départ, ce qui suggère un parcours circulaire de sa promenade. Claudel exprime le besoin de s'assurer de la réalité de sa perception visuelle par une évidence tactile. D'un geste bizarre donc, le poète ferme les yeux et il tend sa main pour confirmer la présence du mur toujours à sa droite, une évidence de solidité qui contrarie son impression de fragilité, « tout un monde fragile ».

En fait, ce mur pourrait bien faire référence à une muraille vraie dans la ville de Tokyo ou bien évoquer le souvenir de la Grande Muraille en Chine et la mer, éléments du paysage ou Claudel écrit son « Processionnal » en 1907. Cependant, il se peut bien que cette image soit aussi abstraite. La répétition, six fois, de l'expression « à ma droite ", répétition soulignée d'ailleurs par l'adverbe « continuellement " et l'adjectif « indécollable » arrache cette image du domaine de la réalité pour la placer dans une aire 
symbolique. On dirait que cette présence constante, ce mur, symbolise une sorte d'ange gardien, une présence protectrice qui l'accompagne, qui le précède et le suit dans sa route. L'adjectif, « intérieure » dans le titre suggère aussi une réalité abstraite, qui n'est pas tangible.

L'image est reprise avec ces valeurs abstraites à la fin du poème, dans le $12^{\mathrm{e}}$ fragment. Le mur ici devient un « anneau " qui retient le poète à l'extérieur, dans une zone d'exil on dirait. D'ailleurs, le poète désigne le mur ici comme une présence abstraite, un mur qui n'est pas « dehors " mais « dedans » et il en est le prisonnier. Donc la présence plutôt positive protectrice, vigilante, dans la première partie du poème se métamorphose en image plutôt contraignante et même maléfique à la fin. Ce mur lui interdit l'accès au «centre » :

J'ai appris que pour aller d'un point à un autre il est possible de passer partout excepté par le centre.

Claudel fait référence ici, peut-être, aux contraintes de sa vie à l'étranger, à ses déplacements d'un pays a un autre sans même pouvoir passer par le «centre », c'est-à-dire son pays mental. En fait, la $2^{\mathrm{e}}$ et la $3^{\mathrm{e}}$ partie de ce poème évoquent le passage du poète du Brésil au Japon ("Le paysage futur se levait à travers une vapeur blanche...») et sa ligne de vie discontinue, faite de «feuilles interrompues ", de "mondes décollés ". Il passe de l'Occident à l'Orient, contournant l'Europe aux prises de la guerre. C'est un passage dur pour lui «sans aucune espèce d'égard ou de transition », écrit-il. Mais, il voit aussi une sorte de prédestination dans le fragment brésilien de "Sainte Geneviève " dont l'ombre des mots « au verso » de la page annonce pour lui une suite future et une nouvelle composition, c'est-à-dire le poème « La Muraille intérieure de Tokyo ». Cette méditation du poète confirme deux réalités à la fois : celle actuelle et celle passée/future ou intemporelle, celle présente et celle absente, celle du « dehors » et celle du « dedans ", celle existentielle et celle artistique. Les autres fragments, du $4^{\mathrm{e}}$ au $11^{\mathrm{e}}$, approfondissent et élargissent cette méditation 


\section{Sergio Villani}

binaire avec d'autres analogies : un papier blanc, une nappe de neige, un ruban d'eau, le « verre de l'étang », et l'« étoffe du sommeil ». Tout devient matière à capter l'invisible. L'art, le poème, incorpore, incarne, l'invisible dans le visible ou le tangible. Le poème, surtout exemplifie une réalité multidimensionnelle et polysémique, « d'innombrables écritures ». Le faire poétique, poiésis, est une mimésis de la réalité actuelle, tangible et visible, terrestre, mais c'est aussi le reflet d'une réalité intemporelle, spirituelle, celle « au-dessus de notre histoire », écrit Claudel; et aussi le reflet de « l'énorme coulée invisible », celle des générations et des écritures futures :

Je veux écrire un poëme qui invite l'esprit à la fois sur une triple route.

La première est en haut celle des Saints audessus de nous reprenant, recomposant chacun de nos mouvements en une offrande solennelle, leur procession au-dessus de notre histoire.

La seconde est le poëme lui-même comme un torrent de mots, comme une grande rue moderne tout emplie d'une masse de peuple qui marche dans le même sens, chacun libre entre ses voisins.

La troisième de l'autre côté du papier est ce grand fleuve qu'on ne voit pas,

Il faut pour le révéler cette poignée de roseaux tout à coup qui interrompt le courant, ce pétillement de la lune sous le ventre d'une sarcelle,

Ou simplement une mouche à feu et son reflet, cette unique paillette de feu qui révèle l'énorme coulée invisible. (OP, p. 646, fragment $\mathrm{V}$ )

Il est clair que Claudel nous invite à faire une lecture allégorique de son poème, à y voir des couches superposées de signification. L'art est une activité mimétique, reflet non seulement de réalités terrestres, mais aussi de réalités invisibles et intemporelles. 
Poésie et allégorie : « La muraille intérieure de Tokyo »

L'image du mur paraît aussi au début de l'« Ode jubilaire » sur Dante!

Ce mur, avec son dessin toujours le même à un mètre, ses énervantes questions toujours les mêmes, y coller nos belles histoires l'une sur l'autre, c'est difficile de l'empêcher dessous peu de redevenir bizarre et transparent (OP, p. 675)

L'image du mur représente encore ici la réalité terrestre, désignée par Claudel incomplète, partielle et fragmentaire. Il faut l'art et la foi pour révéler d'autres dimensions de cette réalité. Alors, le mur acquiert une apparence inhabituelle, «bizarre » et devient « transparent », véhicule pour révéler une réalité totale, source d'une connaissance complète.

«La muraille intérieure de Tokyo », lu dans le contexte d'autres poèmes de la même époque, comme l' «Ode jubilaire », acquiert une importance singulière, non seulement par son expression allégorique de la vision existentielle et poétique de Claudel mais aussi, et peut-être plus pertinent à l'histoire littéraire, parce qu'il annonce un intérêt, une préoccupation, avec cette image du mur de la part de nombreux écrivains et artistes de l'Après-guerre que ce soit « le mur » de Sartre, « les murs » et la «paroi » de Guillevic ou les graffitis de l'" art brut » de Dubuffet. Cette méditation claudélienne sur la «muraille » génère donc, directement ou indirectement, un « paysage futur », intellectuel et artistique, fait de «feuilles décollées », certes, mais tout à fait d'une intertextualité riche et féconde.

\section{Note}

1 Paul Claudel, Euvre poétique, éd. Jacques petit, Paris : Gallimard, 1967. Abréviation, OP. 